Case Report

\title{
Spontaneous Bacterial Peritonitis and Henoch-Schönlein Purpura in a Patient with Liver Cirrhosis
}

\author{
Neil Gupta, Joyce Kim, and Basile Njei \\ Yale University School of Medicine, 333 Cedar Street, New Haven, CT 06510, USA \\ Correspondence should be addressed to Neil Gupta; neil.gupta@yale.edu
}

Received 23 February 2015; Accepted 15 April 2015

Academic Editor: Eldon A. Shaffer

Copyright (C) 2015 Neil Gupta et al. This is an open access article distributed under the Creative Commons Attribution License, which permits unrestricted use, distribution, and reproduction in any medium, provided the original work is properly cited.

\begin{abstract}
Henoch-Schönlein purpura (HSP) is a small vessel systemic vasculitis, predominantly affecting children, characterized by a tetrad of manifestations, specifically palpable purpura, arthralgia, abdominal pain, and renal disease. HSP in the adult population is rare, and no case has been described of HSP in liver cirrhosis with spontaneous bacterial peritonitis (SBP). We present the case of a 58year-old male with liver cirrhosis, who was subsequently diagnosed with SBP and later HSP. In this patient, the diagnosis of HSP was demonstrated clinically by his palpable purpura, diarrhea, hematuria, and abdominal pain and confirmed pathologically by his renal and skin biopsies demonstrating leukocytoclastic vasculitis and IgA complexes. We believe that this is an example of altered IgA processing in cirrhosis leading to the development of IgA immune complexes and ultimately HSP. The patient additionally had SBP, which may have increased his risk for developing HSP given antigen processing by mucosa-associated lymphoid tissues leading to immune complex deposition, which may not have been effectively cleared in the context of his liver disease. The patient unfortunately died of gastrointestinal hemorrhage, which is unclear to be due to his underlying cirrhosis or a gastrointestinal manifestation of HSP itself.
\end{abstract}

\section{Introduction}

Henoch-Schönlein purpura (HSP) is a small vessel systemic vasculitis, predominantly affecting children, with ninety percent of cases occurring in the pediatric population. HSP is characterized by a tetrad of manifestations, specifically palpable purpura, arthralgia, abdominal pain, and renal disease, with gastrointestinal and renal involvement more prevalent in older adults $[1,2]$. The characteristic finding is leukocytoclastic vasculitis accompanied by IgA immune complexes within affected organs. Gastrointestinal symptoms of HSP can range from mild symptoms of nausea, vomiting, or abdominal pain to gastrointestinal hemorrhage, bowel ischemia, and bowel perforation [3]. About half of cases of HSP are preceded by an upper respiratory infection or other underlying infections. HSP in the adult population is rare, and no case has been described of HSP in liver cirrhosis with spontaneous bacterial peritonitis (SBP). We present the case of a 58-year-old male with liver cirrhosis and SBP, confounded by the diagnosis of HSP.

\section{Case Report}

A 58-year-old male with past medical history of diabetes mellitus type 2, depression, HTN, chronic alcohol abuse, and $\mathrm{BPH}$, presented to our hospital with worsening leftlower quadrant abdominal pain over three weeks and painless hematuria for five days. He had no known history of liver or kidney disease. Physical examination was significant for abdominal distension and left-lower quadrant abdominal tenderness. His initial labs demonstrated pancytopenia (white blood cell count 3.3, hemoglobin 7.7, hematocrit 23.4, and platelets 95), acute kidney injury (creatinine 1.6, unknown baseline), hypoalbuminemia (albumin 3.0), hyponatremia (sodium 129), and a nonanion gap metabolic acidosis (bicarbonate 15.5), Table 1(a). Urine studies showed large amount of blood and RBCs with some hyaline casts and proteinuria, and urine electrolytes showed $\mathrm{FeNa}$ of $0.8 \%$, Table $1(\mathrm{~d})$. CT abdomen showed a nodular margin of the liver with mild intrahepatic biliary dilation, suggesting chronic liver disease, along with splenomegaly. US abdomen 
TABle 1: (a) Initial labs. (b) Other labs. (c) Paracentesis. (d) Urine studies.

(a)

\begin{tabular}{lcc}
\hline Lab & Value & Reference \\
\hline $\mathrm{Wbc}$ & 3.3 & $(4-10)$ \\
$\mathrm{Hg}$ & 7.7 & $(14-18)$ \\
$\mathrm{Hct}$ & 23.4 & $(40-52)$ \\
Platelets & 95 & $(150-350)$ \\
$\mathrm{Na}$ & 129 & $(135-145)$ \\
$\mathrm{K}$ & 3.7 & $(3.5-5)$ \\
$\mathrm{Cl}$ & 98 & $(96-106)$ \\
$\mathrm{HCO}$ & & $(22-30)$ \\
$\mathrm{AG}$ & 15.5 & $(7-16)$ \\
$\mathrm{BUN}$ & 16 & $(7-20)$ \\
$\mathrm{Cr}$ & 26 & $(0.5-1.2)$ \\
$\mathrm{Glucose}$ & 1.6 & $(70-100)$ \\
$\mathrm{AST}$ & 217 & $(0-34)$ \\
$\mathrm{ALT}$ & 49 & $(0-34)$ \\
$\mathrm{ALP}$ & 31 & $(30-130)$ \\
$\mathrm{GGT}$ & 184 & $(11-49)$ \\
$\mathrm{DB}$ & 253 & $(>0.20)$ \\
TB & 0.11 & $(<1.20)$ \\
Albumin & 0.23 & $(3.5-5)$ \\
$\mathrm{INR}$ & 3.0 & $(1-2)$ \\
Ammonia & 0.92 & $(11-35)$ \\
AFP & 72 & $(<6)$ \\
\hline
\end{tabular}

(b)

\begin{tabular}{lcc}
\hline Lab & Value & Reference \\
\hline ANA & $<1: 40$ & $(<1: 40)$ \\
ANCA & negative & \\
Anti-DNASE B ab & $<80$ & $(0-200)$ \\
Antismooth IgG & 13 & $(<20)$ \\
C3 & 86 & $(81-145)$ \\
C4 & 32 & $(16-39)$ \\
Ceruloplasmin & 29 & $(18-51)$ \\
Ferritin & 36 & $(30-400)$ \\
HgA1c & 8.0 & $(4.0-6.0)$ \\
HIV & negative & \\
HbSAg & negative \\
Hep C Ab & negative & \\
Hep BcIgM & negative & \\
Hep A IgM & negative & \\
LDH & 181 & $(118-273)$ \\
Mitochondrial ab & 4.7 & $(<20)$ \\
Ova/parasites urine & negative & $(60.20)$ \\
Schistosoma ab & 0.95 & $(0.27-4.20)$ \\
TSH & 1.62 & \\
Total protein & 5.9 & \\
\hline
\end{tabular}

(c)

\begin{tabular}{lc}
\hline Lab & Value \\
\hline Glucose & $182 \mathrm{mg} / \mathrm{dL}$ \\
\hline
\end{tabular}

(c) Continued.

\begin{tabular}{lc}
\hline Lab & Value \\
\hline LDH & $79 \mathrm{U} / \mathrm{L}$ \\
Protein & $1.7 \mathrm{~g} / \mathrm{dL}$ \\
Albumin & $0.9 \mathrm{~g} / \mathrm{dL}$ \\
RBC & $6400 \mathrm{cells} / \mathrm{uL}$ \\
Nucleated cells & 1850 cells $/ \mathrm{uL}$ \\
Differential & $51 \%$ granulocytes, $17 \%$ \\
& lymphocytes, and 32\% tissue cells \\
Cytology & Negative for malignancy, \\
& mesothelial cells with histiocytes \\
Culture & and neutrophils \\
\hline
\end{tabular}

(d)

\begin{tabular}{lc}
\hline Lab & Value \\
\hline Protein & $100 \mathrm{mg} / \mathrm{dL}$ \\
Blood & Large \\
Bilirubin & Negative \\
Leukocytes & Negative \\
Nitrites & Negative \\
Hyaline casts & 3 \\
WBC/HPF & 17 \\
RBC/HPF & 1363 \\
Urine Na & $31 \mathrm{mmol} / \mathrm{L}$ \\
Urine K & $17.9 \mathrm{mmol} / \mathrm{L}$ \\
Urine Cl & $16 \mathrm{mmol} / \mathrm{L}$ \\
Urine Cr & $50.7 \mathrm{mg} / \mathrm{dL}$ \\
Urine UN & $666 \mathrm{mg} / \mathrm{dL}$ \\
FeNa & $0.8 \%$ \\
\hline
\end{tabular}

confirmed heterogeneous and nodular contour of liver with splenomegaly and moderate amount of ascites, likely sequelae of cirrhosis, with dopplers negative for thrombosis. The nodular liver on CT, thrombocytopenia, hypoalbuminemia, splenomegaly, and moderate ascites all helped establish the new diagnosis of liver cirrhosis.

Despite further labs to exclude viral, autoimmune, and other causes, Table 1(b), the etiology of his liver cirrhosis remained unclear, attributable to NASH versus alcohol given his history of uncontrolled diabetes and chronic etoh abuse, Table 1(b). Diagnostic paracentesis was performed, showing glucose $182, \mathrm{LDH} 79$, albumin 0.9 , and protein 1.7 with 6400 RBCs and 1850 nucleated cells (51\% granulocytes, 17\% lymphocytes, and $32 \%$ tissue cells), Table 1(c). Cytopathological examination revealed no neoplastic cells, and bacterial culture was negative. The corrected neutrophil count of 917 and serum-ascites albumin gradient of 2.1 were consistent with SBP and portal hypertension, Table 1(c).

After diagnosis of liver cirrhosis (Child-Pugh class B, MELD 6) and SBP, he was treated with IV ceftriaxone (2 $\mathrm{g}$ daily) and IV albumin ( $1.5 \mathrm{~g} / \mathrm{kg}$ on first day and $1 \mathrm{~g} / \mathrm{kg}$ on third day). He was placed on protonix given portal hypertension with unknown variceal status and was monitored for signs of hepatic encephalopathy, though he mentated well with no asterixis on exam. Repeat diagnostic paracentesis on day 


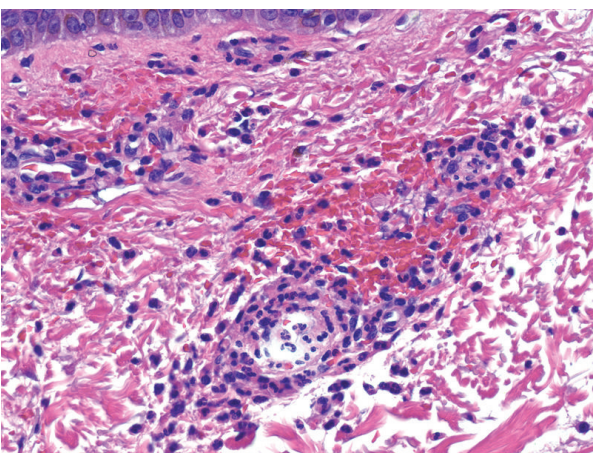

(a)

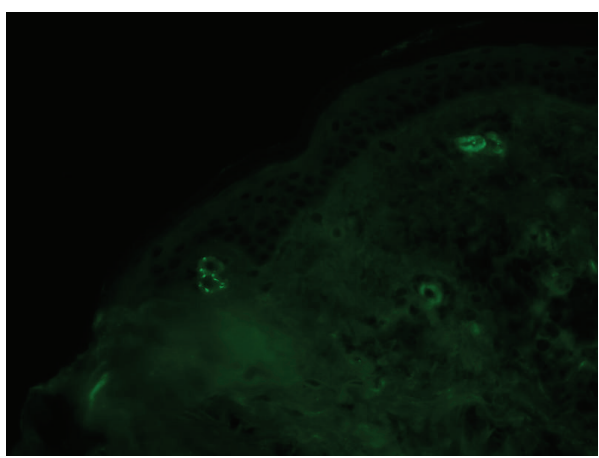

(c)

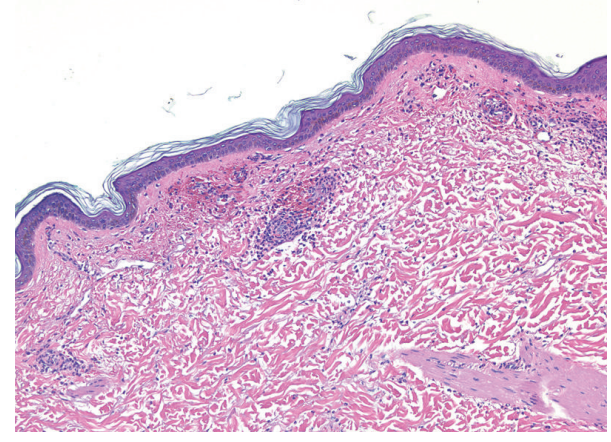

(b)

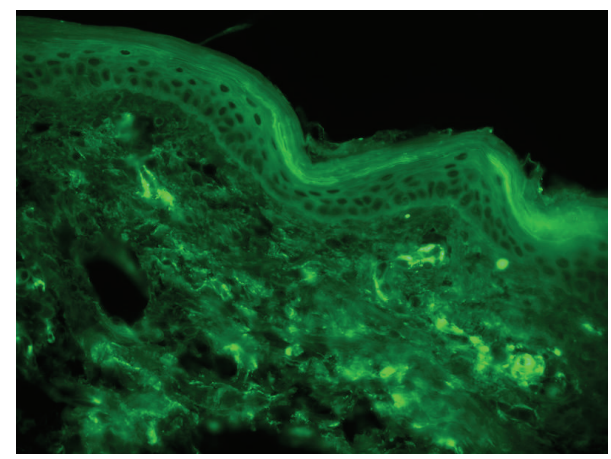

(d)

Figure 1: Skin biopsy, right arm. Top images are H\&E slides of arm at high power (a) and low power (b), showing fibrin, neutrophils, and neutrophil fragments near vessels along with extravasated erythrocytes and a mixed cellular infiltrate. Bottom images are direct immunofluorescence (DIF) showing vascular wall staining with C3 (c) and IgA (d) supporting the diagnosis of HSP.

3 demonstrated resolution of his SBP with 8150 RBCs and 305 nucleated cells (3\% granulocytes, 51\% lymphocytes, $45 \%$ tissue cells, and $1 \%$ eosinophils).

Despite the underlying diagnosis of liver cirrhosis and SBP, the basis of his renal dysfunction and hematuria continued to remain a mystery. Cystoscopy was negative for malignancy, and his urine sediment did not reveal any dysmorphic RBCs. Quantification of his proteinuria revealed a protein: creatinine ratio of 1.9. There was concern for hepatorenal syndrome (HRS) given his low FeNa and bland urine sediment. He was given albumin with no improvement in his renal function in addition to treating underlying SBP, while additional treatments such as midodrine and octreotide were not pursued given his high MAPs. Work-up for other causes of his renal failure continued.

Four days after admission, he had diarrhea, and thirteen days later, he developed new purpuric macules and papules, approximately $0.3-1.5 \mathrm{~cm}$ in size, most prominent on his elbows but also on his abdomen, buttocks, back, and inguinal folds. Skin biopsy showed fibrin, neutrophils, and neutrophil fragments near vessels with mixed cellular infiltrate and extravasated erythrocytes, Figures 1(a) and 1(b). Direct immunofluorescence (DIF) studies showed deposition of IgA and C3 in vessel walls, supporting the diagnosis of HSP, Figures 1(c) and 1(d). Due to worsening renal function with creatinine rising to 4.9 , renal biopsy was performed revealing mesangial proliferative glomerulonephritis,
Figures 2(a) and 2(b), with crescents, Figures 2(c) and 2(d), and IgA, Figure 2(e), also consistent with HSP. He was given one dose of solumedrol $500 \mathrm{mg}$ IV and started on prednisone $60 \mathrm{mg}$, which was tapered to $50 \mathrm{mg}$ daily, with protonix to decrease GI bleeding risk. The purpuric lesions subsequently resolved with corticosteroid treatment.

However, his renal failure continued to worsen with his creatinine rising to 5.1 and BUN to 157. Due to concern for worsening renal failure, uremia, and oliguria, he underwent emergent hemodialysis. His course quickly deteriorated as he became acutely hypotensive, with a sudden hemoglobin drop to 4.8. After two units of transfusion to hemoglobin of 8.8, he coded one day later with profuse hematemesis after an episode of bright red blood per rectum and died.

\section{Discussion}

In this patient, the diagnosis of HSP was demonstrated clinically by his palpable purpura, diarrhea, hematuria, and abdominal pain. The diagnosis was confirmed pathologically by his renal and skin biopsies demonstrating leukocytoclastic vasculitis and IgA complexes. While HSP is predominantly a pediatric disorder, its existence in adults is rare and its association with liver cirrhosis is even more scant [4-6]. There are only a few case reports describing the association between liver cirrhosis and HSP. For example, Aggarwal et al. reported one of the first cases of liver cirrhosis with acute renal failure 


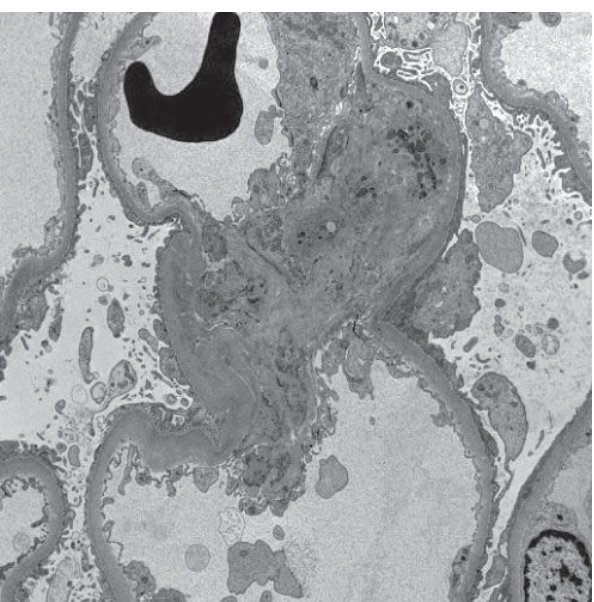

(a)

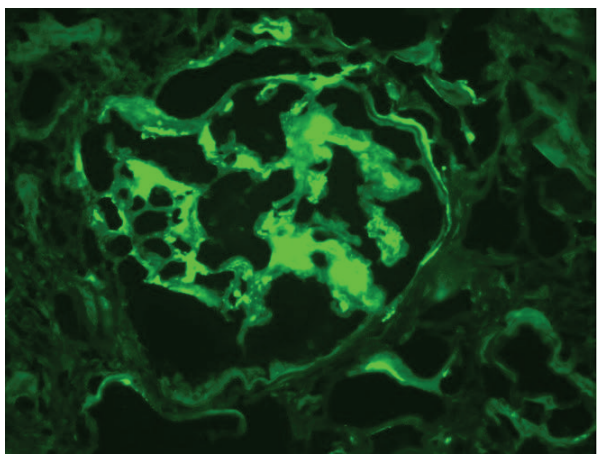

(c)

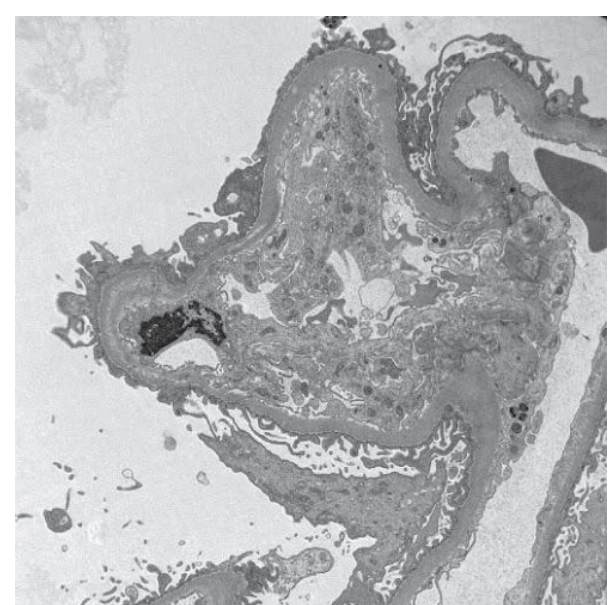

(b)

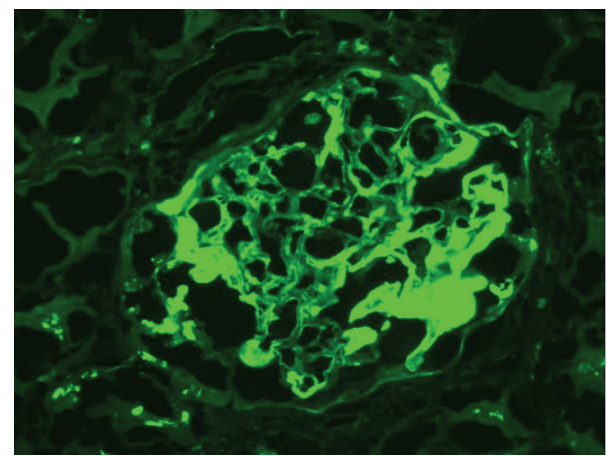

(d)

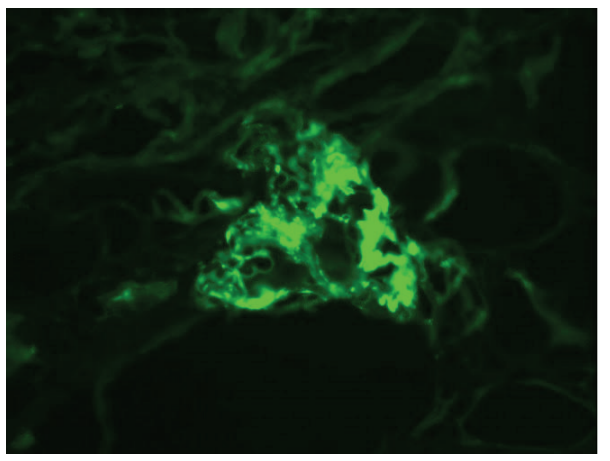

(e)

FIGURE 2: Renal biopsy. Top images ( $\mathrm{a}$ and $\mathrm{b}$ ) are electron microscopy slides demonstrating increase in mesangial matrix with segmental mesangial deposits. The glomerular architecture shows corrugation of basement membrane with focal effacement of foot processes. Bottom images are direct immunofluorescence (DIF) showing glomerular staining with C3 (c), fibrinogen (d), and IgA (e), supporting diagnosis of HSP.

and HSP [4]. Our case, however, is the first to show the significant extent of HSP in liver cirrhosis due to the combined renal and skin manifestations along with the additional complicating factor of SBP, which may have triggered his course of HSP.

Alcohol liver disease is characterized by IgA deposits in a continuous pattern along liver sinusoids, in addition to skin capillaries and mesangium of renal glomeruli. The liver contributes to the clearance of intravascular IgA, causing patients with chronic liver disease to show an increase in serum IgA concentration [7]. Van de Wiel et al. studied the presence and concentration of circulating IgA-containing immune complexes in patients with alcoholic liver disease and patients with other nonalcoholic liver diseases with comparable serum IgA levels [8]. He concluded that the presence of circulating IgA-containing immune complexes was directly related to the severity of liver damage and substantiated the pivotal role of the liver in clearing circulating IgA [8]. The role of liver cirrhosis in the development of HSP is intriguing since this patient's chronic liver disease may have precipitated the development of HSP with defective liver 
metabolism of IgA circulating immune complexes, leading to deposition in the skin and kidneys.

The diagnosis of SBP is particularly unique in this case and has not been previously reported in case reports of patients with combined HSP and liver cirrhosis. Observations in children with HSP have confirmed approximately 30$65 \%$ of IgA vasculitis cases occur after an upper respiratory tract infection [9]. Some propose that increased synthesis of IgA due to antigen processed by the mucosa-associated lymphoid tissue leads to development of the disease by immune complex deposition between antigens and $\operatorname{IgA}$ in the skin, gut, and kidneys [9]. In this particular case, it could be hypothesized that SBP may have triggered antigen processing by mucosa-associated lymphoid tissue in the gut and the subsequent development of IgA complexes, with impaired clearing due to the patient's underlying liver cirrhosis, leading to the significant extent of his HSP.

This patient unfortunately had a rapid decline due to his hospital stay. The extent of his HSP with combined renal and skin manifestations has previously not been described in the literature in association with liver cirrhosis. While he received corticosteroid treatment, this does not prevent nephritis or alter the course of HSP $[10,11]$. His renal failure may have also been worsened by SBP and underlying cirrhosis, in addition to his IgA nephropathy. His ultimate hematemesis may have been due to variceal hemorrhage in the context of his liver cirrhosis, though gastrointestinal hemorrhage is known to be a rare manifestation of HSP.

\section{Conclusions}

This is a unique case of significant HSP with both renal and skin manifestations along with SBP in a patient with underlying liver cirrhosis. While HSP is rare in adults, we believe that this is an example of altered IgA processing in cirrhosis leading to the development of IgA immune complexes and ultimately HSP [7]. The patient additionally had SBP, which may have increased his risk for developing HSP given antigen processing by mucosa-associated lymphoid tissues leading to immune complex deposition, which may not have been effectively cleared in the context of his liver disease. The patient unfortunately died of gastrointestinal hemorrhage, which is unclear to be due to his underlying cirrhosis or a gastrointestinal manifestation of HSP itself.

\section{Conflict of Interests}

The authors declare that there is no conflict of interests regarding the publication of this paper.

\section{Acknowledgments}

The authors first acknowledge St. Raphael's Campus of Yale New Haven Hospital including all medical and nursing staff for the care of this patient. They would also like to acknowledge Dr. Shawn Cowper, Associate Professor of Dermatology and Pathology; Dr. Rob Munday, Pathology Resident; and Dr. Gilbert Moeckel, Associate Professor of
Pathology and Director of Renal Pathology and Electron Microscopy Laboratory of the Yale School of Medicine for their assistance with the pathology slides.

\section{References}

[1] R. Blanco, V. M. Martínez-Taboada, V. Rodríguez-Valverde, M. García-Fuentes, and M. A. González-Gay, "Henoch-Schonlein purpura in adulthood and childhood: two different expressions of the same syndrome," Arthritis \& Rheumatism, vol. 40, no. 5, pp. 859-864, 1997.

[2] C. Garcia-Porrua and M. A. Gonzalez-Gay, "Comparative clinical and epidemiological study of hypersensitivity vasculitis versus Henoch-Schönlein purpura in adults," Seminars in Arthritis \& Rheumatism, vol. 28, no. 6, pp. 404-412, 1999.

[3] J. C. N. Chan, P. K. T. Li, F. M. Lai, and K. N. Lai, "Fatal adult Henoch-Schonlein purpura due to small intestinal infarction," Journal of Internal Medicine, vol. 232, no. 2, pp. 181-184, 1992.

[4] M. Aggarwal, C. L. Manske, P. J. Lynch, and M. S. Paller, "Henoch-Schonlein vasculitis as a manifestation of IgAassociated disease in cirrhosis," The American Journal of Kidney Diseases, vol. 20, no. 4, pp. 400-402, 1992.

[5] M. Ogawa, Y. Makino, S. Ueda, M. Ohto, and B. Akikusa, "Rapidly progressive glomerulonephritis in association with Henoch-Schonlein purpura in a patient with advanced liver cirrhosis," Nephron, vol. 71, no. 3, pp. 365-366, 1995.

[6] R. Robeva, R. Krŭsteva, and N. Belovezhdov, "Benign monoclonal gammopathy in a female patient with Schönlein-Henoch glomerulonephritis and liver cirrhosis," Vŭtreshni Bolesti, vol. 29, no. 5, pp. 105-109, 1990.

[7] G. C. Newell, "Cirrhotic glomerulonephritis: incidence, morphology, clinical features and pathogenesis," American Journal of Kidney Diseases, vol. 9, no. 3, pp. 183-190, 1987.

[8] A. Van de Wiel, R. M. Valentijn, H.-J. Schuurman, M. R. Daha, R. J. Hene, and L. Kater, "Circulating IgA immune complexes and skin IgA deposits in liver disease. Relation to liver histopathology," Digestive Diseases and Sciences, vol. 33, no. 6, pp. 679-684, 1988.

[9] M. Piram and A. Mahr, "Epidemiology of immunoglobulin A vasculitis (Henoch-Schönlein): current state of knowledge," Current Opinion in Rheumatology, vol. 25, no. 2, pp. 171-178, 2013.

[10] G. B. Fogazzi, S. Pasquali, M. Moriggi et al., "Long-term outcome of Schonlein-Henoch nephritis in the adult," Clinical Nephrology, vol. 31, no. 2, pp. 60-66, 1989.

[11] I. S. Szer, "Gastrointestinal and renal involvement in vasculitis: management strategies in Henoch-Schonlein purpura," Cleveland Clinic Journal of Medicine, vol. 66, no. 5, pp. 312-317, 1999. 


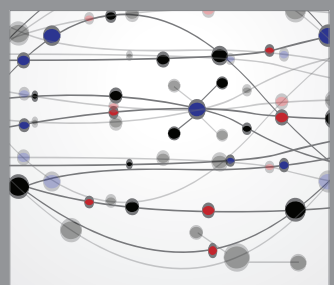

The Scientific World Journal
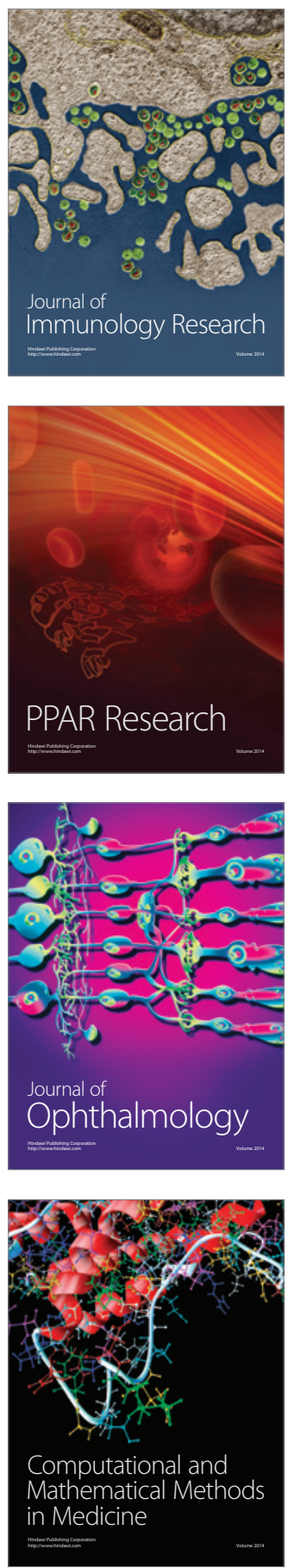

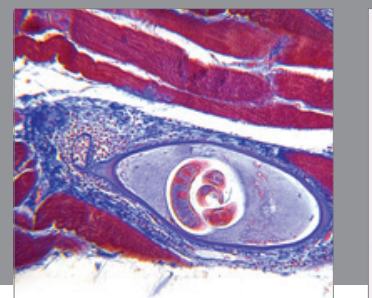

Gastroenterology

Research and Practice
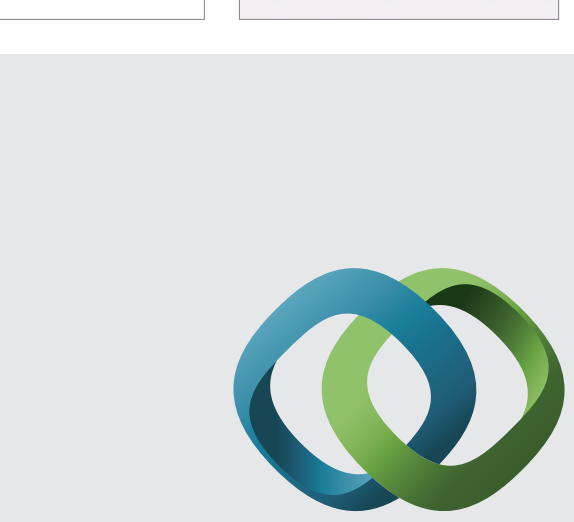

\section{Hindawi}

Submit your manuscripts at

http://www.hindawi.com
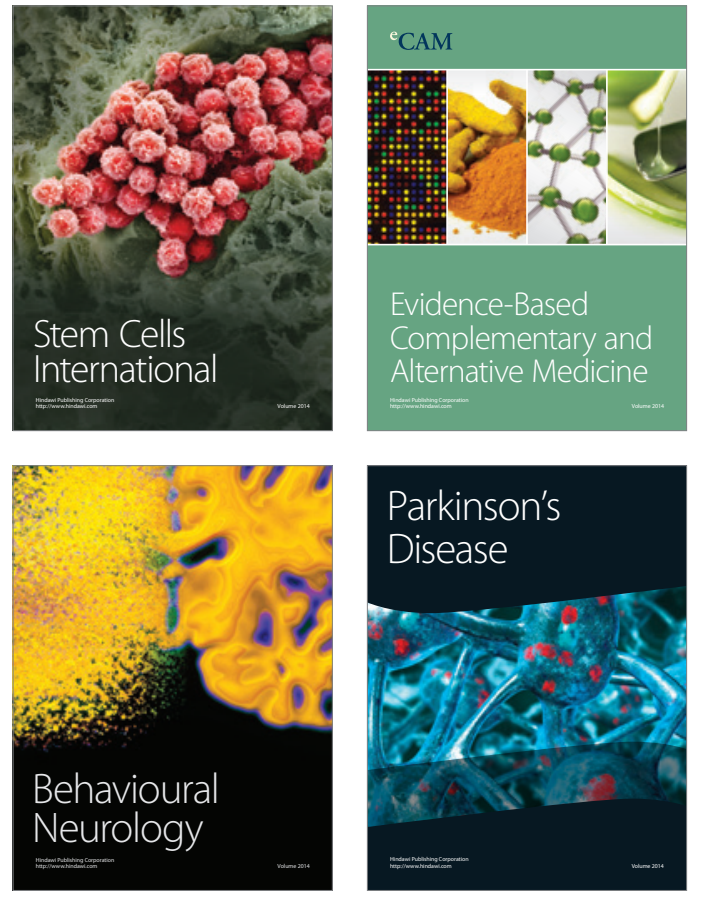
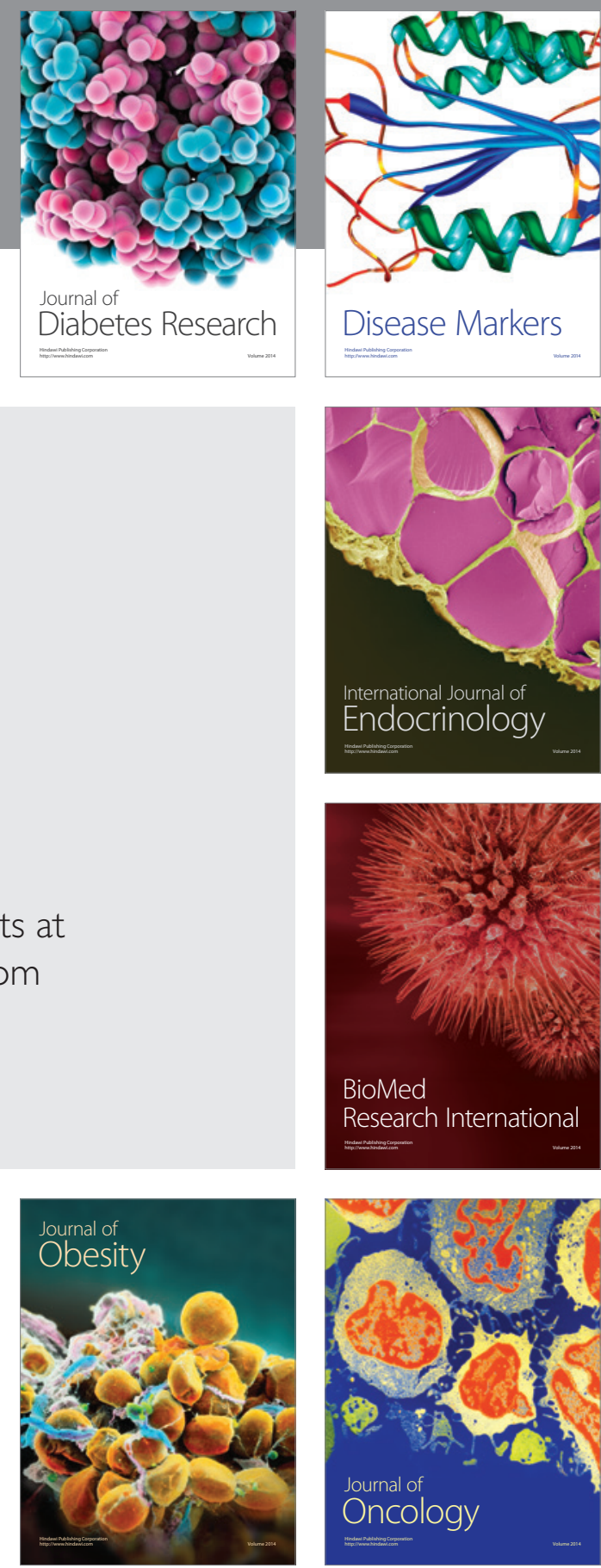

Disease Markers
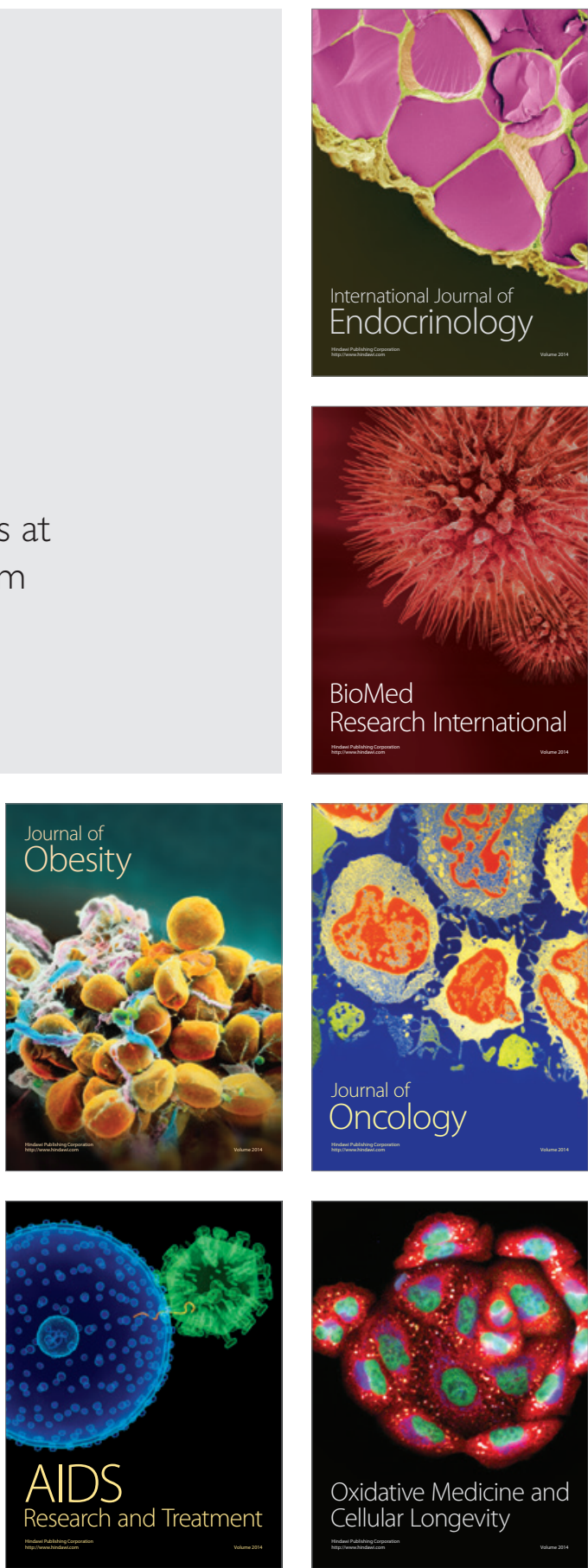